\title{
The Swedish Twin Registry in the Third Millennium
}

\author{
Nancy L. Pedersen 1,2, Paul Lichtenstein', and Pia Svedberg \\ 'Department of Medical Epidemiology, Karolinska Institutet, Sweden \\ ${ }^{2}$ Department of Psychology, University of Southern California, USA
}

Sin ince the Swedish Twin Registry was first established in the late 1950s to study the importance of smoking and alcohol consumption on cancer and cardiovascular diseases, it has been expanded and updated on several occasions. The focus has similarly broadened to most common complex diseases. The content of the database is described, ongoing projects based on the registry are summarized, and we review some of the principal findings on aging, cancer and cardiovascular disease that have come from the registry. Ongoing efforts and future plans for the STR are discussed. Among others, we plan blood collection and genotyping to study the genetic bases of complex diseases, a first contact ever with the cohorts born after 1958, and in-depth studies of selected diseases, such as Parkinson's disease and chronic fatigue syndrome.

The Swedish Twin Registry (STR) is a unique resource for the scientific community. It was first established in the late 1950s to study the importance of smoking and alcohol consumption on cancer and cardiovascular diseases while controlling for genetic propensity to disease. Since that time, the STR has been expanded and updated on several occasions, and the focus has similarly broadened to most common complex diseases. A more thorough description of the STR and a review of results have recently been published elsewhere (Lichtenstein et al., 2002).

\section{Recruitment}

There are three cohorts in the registry, each of which differs in method of ascertainment and extent of data collected. Cederlöf and Lorich (1978) described the first two cohorts, and in the following, we describe the current structure of the registry. The vital status of the pairs in the registry, as of January 1, 2002, is summarized in Table 1.

\section{The Cohort Born 1886-1925}

When the twin registry started in 1959, all parishes in Sweden were contacted to obtain information concerning multiple births between 1886 and 1925 (Cederlöf, 1966). Each potential pair of twins was manually followed until status in 1959 was established. In 1960-61, a mail-out questionnaire was sent to all like-sexed twins where both were alive and living in Sweden. Since then, questionnaires have been sent out in 1963 and 1967, with complementary questionnaires sent to a subset in 1970. The information is mainly of demographic, medical and lifestyle character, with special attention to cardiovascular and respiratory disease, general health, legal drug use, diet, and psychosocial conditions.

\section{The Cohort Born 1926-1958}

In 1970 a new cohort of twins born 1926-1967 was compiled, this time by use of nationalized birth registrations. A birth register consisting of all 50,000 twin births was established (Medlund et al., 1977). Members of like-sexed pairs from the cohort born 1926-1958 were sent a mail-out questionnaire in 1972-1973. Responses were received from about 36,000 individuals in 14,000 twin pairs (Medlund et al., 1977). This questionnaire was very similar to those sent to the older cohort. In addition, items from the Eysenck Personality Inventory and a series of questions about environmental irritants were included. Information has been maintained concerning both the initial birth cohort as well as the subsample of like-sexed pairs from which the questionnaire information was obtained.

\section{The Cohort Born 1959-1990}

Twins born 1959-1968 had already been identified in the birth register when the 1926-1958 cohort was compiled, but were not contacted. In 1993, twins born 1969-1990 were included by record linkage to the Medical Birth Registry. From this cohort, only the parents of twin pairs born between 1985 and 1986 have been contacted (Lichtenstein \& Svartgren, 1997). Because no contact has been made, we have no information regarding zygosity of the like-sexed pairs except those born 1985-1986.

\section{Registry Linkages}

The STR is regularly matched to national health care registries to obtain information on cancer diagnoses, inpatient discharges, causes of death, conditions during birth, and current address and vital status.

\section{Current Major Research Focus}

At present, there are several ongoing projects based on the STR covering a broad spectrum of public health domains. Some of the areas being investigated are behavioral problems, aging, dementia, allergy, cancer, gastrointestinal, psychiatric and cardiovascular disease.

\section{Screening Across the Lifespan Twin study (SALT)}

The Swedish Twin Registry is currently in the final phase of a complete screening of all twins born 1958 or earlier

Address for correspondence: Nancy L. Pedersen, Department of Medical Epidemiology, Karolinska Institutet, Box 281, SE 17177 Stockholm, Sweden. Email: Nancy.Pedersen@mep.ki.se 
Table 1

Vital Status of Individuals in the Swedish Twin Registry as of January 1, 2002, by Birth Year, Zygosity and Sex

(No Information on Zygosity is Available for Most Twins Born After 1958 as They Have Not Yet Been Contacted by the Registry)

\begin{tabular}{|c|c|c|c|c|c|c|c|c|}
\hline \multirow{3}{*}{ Birth year } & \multicolumn{8}{|c|}{ Alive and reachable } \\
\hline & \multicolumn{2}{|c|}{$\mathrm{MZ}$} & \multicolumn{2}{|c|}{ DZ } & \multicolumn{2}{|c|}{$0 Z$} & \multicolumn{2}{|c|}{$X Z$} \\
\hline & M & $\mathrm{F}$ & $\mathrm{M}$ & $\mathrm{F}$ & M & $\mathrm{F}$ & $\mathrm{M}$ & $\mathrm{F}$ \\
\hline 1886-1890 & 0 & 0 & 0 & 0 & 0 & 0 & 0 & 0 \\
\hline 1891-1895 & 0 & 0 & 0 & 1 & 0 & 0 & 0 & 0 \\
\hline $1896-1900$ & 0 & 1 & 0 & 7 & 0 & 0 & 0 & 0 \\
\hline 1901-1905 & 3 & 9 & 1 & 25 & 0 & 0 & 0 & 1 \\
\hline 1906-1910 & 26 & 96 & 48 & 123 & 23 & 34 & 0 & 4 \\
\hline 1911-1915 & 104 & 208 & 153 & 421 & 113 & 157 & 10 & 9 \\
\hline $1916-1920$ & 233 & 435 & 381 & 808 & 305 & 396 & 6 & 19 \\
\hline $1921-1925$ & 334 & 558 & 658 & 1055 & 546 & 605 & 20 & 26 \\
\hline $1926-1930$ & 452 & 612 & 805 & 1089 & 912 & 1117 & 241 & 313 \\
\hline 1931-1935 & 520 & 667 & 912 & 1086 & 1055 & 1133 & 251 & 265 \\
\hline 1936-1940 & 683 & 814 & 1153 & 1273 & 1279 & 1340 & 268 & 233 \\
\hline 1941-1945 & 1029 & 1205 & 1524 & 1747 & 1737 & 1777 & 446 & 339 \\
\hline 1946-1950 & 1010 & 1145 & 1580 & 1690 & 1843 & 1849 & 603 & 434 \\
\hline 1951-1955 & 933 & 1031 & 1571 & 1462 & 1707 & 1724 & 388 & 260 \\
\hline 1956-1960 & 897 & 1013 & 1529 & 1435 & 1704 & 1722 & 468 & 304 \\
\hline 1961-1965 & 543 & 618 & 917 & 859 & 1513 & 1547 & 1221 & 1139 \\
\hline 1966-1970 & 28 & 25 & 16 & 29 & 1598 & 1583 & 2838 & 2792 \\
\hline 1971-1975 & 14 & 10 & 30 & 24 & 1344 & 1350 & 2843 & 2741 \\
\hline 1976-1980 & 16 & 20 & 16 & 11 & 1072 & 1079 & 2863 & 2760 \\
\hline 1981-1985 & 20 & 14 & 20 & 16 & 1051 & 1039 & 2638 & 2682 \\
\hline 1986-1990 & 35 & 42 & 44 & 38 & 2750 & 2757 & 6848 & 6756 \\
\hline \multirow[t]{2}{*}{ Total } & 6880 & 8523 & 11,358 & 13,199 & 20,552 & 21,209 & 21,952 & 21,077 \\
\hline & \multicolumn{8}{|c|}{ Dead or un-reachable* } \\
\hline \multirow[t]{2}{*}{ Birth year } & \multicolumn{2}{|c|}{$\mathrm{MZ}$} & \multicolumn{2}{|c|}{$\mathrm{DZ}$} & \multicolumn{2}{|c|}{$0 Z$} & \multicolumn{2}{|c|}{$X Z$} \\
\hline & M & $\mathrm{F}$ & M & $\mathrm{F}$ & M & $\mathrm{F}$ & $\mathrm{M}$ & $\mathrm{F}$ \\
\hline $1886-1890$ & 144 & 162 & 156 & 294 & 0 & 0 & 14 & 20 \\
\hline 1891-1895 & 236 & 262 & 280 & 479 & 0 & 0 & 16 & 50 \\
\hline $1896-1900$ & 266 & 333 & 444 & 661 & 0 & 0 & 56 & 38 \\
\hline 1901-1905 & 391 & 467 & 705 & 925 & 0 & 0 & 50 & 77 \\
\hline 1906-1910 & 452 & 504 & 900 & 1015 & 85 & 73 & 56 & 54 \\
\hline 1911-1915 & 474 & 486 & 922 & 897 & 178 & 132 & 40 & 47 \\
\hline 1916-1920 & 383 & 323 & 765 & 632 & 229 & 140 & 46 & 35 \\
\hline $1921-1925$ & 292 & 210 & 580 & 407 & 204 & 145 & 34 & 14 \\
\hline $1926-1930$ & 164 & 111 & 298 & 187 & 454 & 242 & 194 & 114 \\
\hline 1931-1935 & 90 & 83 & 173 & 164 & 232 & 151 & 137 & 66 \\
\hline 1936-1940 & 86 & 67 & 139 & 103 & 197 & 131 & 84 & 55 \\
\hline 1941-1945 & 95 & 71 & 128 & 137 & 216 & 163 & 107 & 62 \\
\hline 1946-1950 & 66 & 59 & 112 & 94 & 118 & 105 & 84 & 55 \\
\hline 1951-1955 & 48 & 47 & 113 & 65 & 121 & 107 & 73 & 58 \\
\hline $1956-1960$ & 46 & 46 & 109 & 64 & 121 & 107 & 78 & 60 \\
\hline 1961-1965 & 23 & 42 & 46 & 42 & 101 & 68 & 85 & 49 \\
\hline 1966-1970 & 3 & 1 & 0 & 5 & 72 & 86 & 178 & 160 \\
\hline 1971-1975 & 2 & 0 & 0 & 4 & 146 & 140 & 375 & 379 \\
\hline 1976-1980 & 0 & 0 & 0 & 1 & 138 & 131 & 449 & 420 \\
\hline 1981-1985 & 2 & 0 & 0 & 0 & 96 & 108 & 274 & 294 \\
\hline 1986-1990 & 1 & 0 & 0 & 2 & 166 & 159 & 442 & 400 \\
\hline Total & 3264 & 3274 & 5870 & 6178 & 2874 & 2188 & 2872 & 2507 \\
\hline
\end{tabular}

Note: ${ }^{*}=$ un-registered, emigrated, classified.

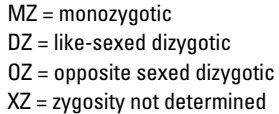


regardless of gender composition or vital status of the pair $(N=52,080)$. This effort is known as SALT: Screening Across the Lifespan Twin study. In March 1998, full scale screening of the twins born 1958 or earlier was initiated. Data collection was performed with a computer assisted telephone interview including a number of items asked to all twins regarding zygosity (similarity), contact with twin partner, family constellation, birth order and weight, different diseases and symptoms, prescription and non-prescription medication use, and permission to collect medical records. All twins are also interviewed regarding their occupation, education, and consumption of alcohol, tobacco, and caffeine, whereas presentation of some items is gender and age specific (e.g., women are asked about hormone replacement therapy and only twins aged 65 years and older are screened for memory problems). The twin's interest in participating in further studies involving a health examination and interview is also solicited. If the twin is unable to be interviewed, the interview is conducted with an informant. All twins in the cohort will have been contacted by September 2002 (Lichtenstein et al., 2002).

Several in-depth studies are ongoing for twins identified through the SALT-study. These include the Study of Dementia in Swedish Twins, Environmental Factors for Parkinson's in Swedish Twins, and a Twin Study of Chronic Fatigue Syndrome in Sweden (see below). Other planned studies with further data collection include studies of rheumatoid arthritis, periodontal disease, eating disorders, depression and anxiety.

\section{Swedish Adoption/Twin Study of Aging - SATSA}

The SATSA sample is a well-defined subset of the Swedish Twin Registry, including all twins who were reared apart and a matched sample of twins reared together (1922 pairs) (Pedersen et al., 1991). The SATSA sample is currently being studied with regard to normal and successful aging through a series of up to 5 questionnaires mailed every 3 years to all living participants as well as 5 waves of inperson cognitive testing and health examinations at rolling 3 year intervals. Questionnaires include measures of physical and psychological health and wellbeing, personality, cognitive status, life events, family environment etc.

\section{The Study of Dementia in Swedish Twins - HARMONY}

The Study of Dementia in Swedish Twins, known by the acronym HARMONY, focuses on genetic and environmental factors for Alzheimer's disease and other dementias. A pilot study based on the SATSA sample involved screening all living members of that cohort born before 1935 regardless of their participation in SATSA testing (Gatz et al., 1997). The HARMONY study now includes all twins in the registry 65 years of age or older identified through SALT as having some indication of cognitive impairment. A total of 13,519 individuals over the age of 65 completed the telephone interview (response rate $=75.9 \%$ ). Dementia screening was based on the TELE, which includes the 10-item MSQ, other cognitive items (counting backwards, recalling three words, and similarities), and questions about health and daily functioning; or on Blessed scores obtained from a proxy interview. Among those screened, 1565 were positive for suspicion of dementia and were referred for complete clinical evaluation by a physician and a nurse. Once a preliminary in-person evaluation suggested that the suspected case was demented, the twin partner was also invited for a clinical work-up. Non-demented twins will be followed up 3 years after the first wave of testing.

\section{ост0-Twin}

OCTO-Twin ("Origins of Variance in the Oldest Old") is a longitudinal study of aging in the oldest-old (McClearn et al., 1997). All like-sexed Swedish twin pairs 80 years old and older, where both in the pair were alive at baseline, were recruited to participate in the study which features 5 longitudinal measurement occasions at 2 year intervals. At wave 1 , both members of 351 pairs participated. Data collection includes questionnaire and health assessments, and extensive cognitive testing in the subjects' homes. Of primary interest are factors contributing to continued wellbeing, health, and functional capacity. Dementia cases are also being identified, building on the methods in the Study of Dementia in Swedish Twins.

\section{Gender}

Gender differences in aging are the focus of the Gender study, which is based on a sample of opposite sexed twins born between 1906 and 1925 (Gold et al., 2002). Because computerized information concerning these pairs was not maintained when the oldest cohort in the registry was compiled in 1961, we manually re-entered information from parish offices and matched birth names and places to the population registry. A total of 1699 potential twin pairs were identified and a questionnaire was sent these pairs in the 1994. Two waves of in-person testing with a 3 year inter-test interval, containing assessments much like those in SATSA and OCTO-Twin have been completed.

\section{Young Twins}

Twins born 1985 and 1986 are being investigated concerning health and behavioural problems. This sample has been followed longitudinally and data from parents, children, and their teachers have been collected on several occasions (Eley et al., 2002; Lichtenstein \& Svartgren, 1997).

\section{Twin Families}

In this study of female twins, their spouses, and their adolescent children, the aim is to study maternal adjustment and family processes and their influence on mental health. Three hundred twenty-six female pairs and their families are included. The families are interviewed and discussions between dyads within the family are also videotaped (Reiss et al., 2001). An extension of this study to male pairs is planned.

\section{Environmental Factors for Parkinson's in Swedish Twins}

Through SALT screening, a large sample of twins have been identified who have either reported that they have been diagnosed with Parkinson's disease, who are taking Parkinson's medications, or who have indicated that they experienced Parkinson-like symptoms that correspond to diagnostic criteria for the disease. These twins and their co-twins are being recontacted to obtain in depth, lifetime exposure information and for clinical and neurological assessments for diagnostic confirmation. Approximately 400 pairs of twins in which one or both members have Parkinson's disease or Parkinson-like symptoms will participate. 


\section{Chronic Fatigue Syndrome}

A substantial number of twins (over 1500) have indicated in SALT screening that they experience symptoms that may be indicative of Chronic Fatigue Syndrome. We are reviewing the medical records of these twins and their partners. After a revised diagnosis is obtained, we will evaluate the genetic architecture of CFS, including comorbidity with fibromyalgia and depression.

\section{Major Achievements CVD}

The list of publications from the STR on cardiovascular disease outcomes and risk factors is extensive (http:// www.mep.ki.se/twin/publications_en.html). Many of the analyses have focused on estimating heritability for cardiovascular endpoints e.g., Heller et al., 1993; Heller et al., 1994; Hong et al., 1997; while others have focused on cardiovascular mortality (Marenberg et al., 1994; Zdravkovic et al., submitted). Hong et al. (1997) demonstrated that the five principal components contained in the insulin resistance syndrome (insulin resistance, body mass index, triglycerides, HDL-cholesterol, and systolic blood pressure), were influenced by a genetic factor in common to the measures, whereas three of the components (triglycerides, insulin resistance, and HDL-cholesterol) were associated with each other due to individual-specific environmental factors (Hong et al., 1997).

In an extended mortality follow-up based upon 26 years of follow-up (1962-1987), 2810 deaths from coronary heart disease and 949 deaths from stroke occurred (Marenberg, 1994). The cumulative probability of survival given the index twin already had died of coronary heart disease was less in $\mathrm{MZ}$ than in DZ twins. With increasing age of the index twin at the time of death from coronary heart disease, the relative hazards decreased continuously for both $M Z$ and $D Z$ twins, and the ratio between $M Z$ and DZ twins converged towards 1 . The relative hazards were only marginally influenced by other risk factors for coronary heart disease. These data clearly show the impact of genetic factors on death from coronary heart disease.

\section{Birth Weight and Acute Myocardial Infarction}

In a case-control study of birth weight retrieved from birth records and acute myocardial infarction (AMI), 132 likesexed twin pairs discordant for AMI and 118 individually-matched control twin pairs were identified (Hubinette et al., 2001). In comparisons between AMI cases and external matched control twins, cases had significantly lower mean birth weight, birth length, and head circumference than controls. In within-pair comparisons between AMI cases and healthy co-twins, no significant differences in birth measurements were found. This lack of an association between birth characteristics and AMI within twin pairs suggests that previously reported associations may be influenced by genetic and early environmental factors.

\section{Aging}

The programs in gerontological genetics based on SATSA, OCTO-Twin and the Gender study have provided a greater understanding of the nature of individual differences in the aging process. Analyses have focused on cognitive abilities, personality, and health outcomes. That genetic influences are still of importance for most behavioral endpoints late in life is one important conclusion from these studies (Pedersen et al., 1996). Comparisons of cross-sectional and longitudinal analyses of SATSA and OCTO-Twin indicate that the importance of genetic effects for general cognitive abilities ("g") is relatively high among the "young-old" (50-70 years of age), but genetic variance decreases longitudinally in those over 70 years of age (Finkel et al., 1998). Heritability for "g" decreases from approximately $80 \%$ in the young-old (Pedersen et al., 1992) to 60\% in the oldestold (McClearn et al., 1997). Findings on dementia indicate that the heritability for Alzheimer's disease is substantial (up to 75\%) (Gatz et al., 1997), but decreases when onset is after the age of 80 (Pedersen et al., 2002).

\section{Cancer in Twins}

Data on 44,788 pairs of twins listed in the Swedish, Danish, and Finnish twin registries were combined in order to assess the risks of cancer at 28 anatomical sites for the co-twins of probands with cancer (Lichtenstein et al., 2000). We found increased risks among the co-twins of affected probands for stomach, colorectal, lung, breast, and prostate cancer. Statistically significant effects of heritable factors were found for prostate cancer $(42 \%)$, colorectal cancer $(35 \%)$, and breast cancer $(27 \%)$. We concluded that inherited genetic factors make a minor contribution to susceptibility to most types of neoplasms, indicating that the environment has the principal role in causing sporadic cancer. The relatively large effect of heritability in cancer at a few sites suggests major gaps in our knowledge of the genetics of cancer.

\section{Future Plans}

The Swedish Twin Registry has recently received stable funding from the government, the pharmaceutical industry as well as from the European Union (together with other European twin registries). This means that we are in the position to initiate new large projects. We are planning to launch several of them the in coming years.

\section{First Contact with 20,000 Pairs of Twin Children}

Previously there has been no registry-wide contact with children through the STR, except for the "Young twins" study (Eley et al., 1999; Lichtenstein \& Annas, 2000; Lichtenstein $\&$ Svartgren, 1997) and the SALT-pilot, where we contacted parents to 150 twin pairs aged 5-17 years in order to test the feasibility of gathering information through a computer assisted telephone interview (Lichtenstein et al., 2002). The pilot study was successful and we now plan to interview about 20,000 parents to all twins in this age range in Sweden. The study will collect information on all major mental and physical health problems including asthma and allergies, headache, autism and autism spectra disorder, and ADHD. Exposure information on substance use and physical activity will also be collected. We also plan to use the study to identify twin pairs of special interested for further phenotyping and/or genotyping in clinical substudies. 


\section{First Contact with Twins Born 1959-1985}

Twins born 1959-1985 have never been contacted by the STR, except for some pairs who have participated in substudies. This cohort of about 23,000 twin pairs will also be contacted, in order to screen for major complex diseases. Again, we will ask for common diseases, symptoms, and medication use as well as for important exposure information (occupation, education, physical activity, diet, alcohol, tobacco, and caffeine). During 2003 we will pilot different data collection strategies for this cohort. Telephone interviews, questionnaires, and/or web-based questionnaires will be tested for their relative efficiency, response rate, and data quality.

\section{Blood Collection and DNA-Analyses}

In order to study the genetic bases of human complex disease the STR, together with the Danish, Dutch, Finnish, Italian, and Norwegian twin cohorts, has received a grant from the European Union. In this study we will collect biological specimens on sub-samples of twin cohorts selected for specific traits (stature, BMI, migraine, coronary heart disease and stroke). The goals are: 1) to utilize maximally the unique features of twin cohorts, including the availability of longitudinal data and ample information about lifestyle and environmental factors, in the characterisation of complex traits; 2) to develop novel mathematical strategies to combine information on genetic profiles underlying common traits (multiple genes and their various alleles with different impacts on disease outcome); 3) to estimate the role of genetic and environmental factors in the disease process in selected traits using dizygotic (DZ) and MZ twins and population cohorts; and 4) to identify major genetic factors predisposing to migraine, coronary heart disease and stroke in European populations.

\section{Augmenting the STR with Information on Birth Characteristics}

It has been suggested that intrauterine hormonal exposures may influence subsequent risks of hormone-dependent cancers among offspring. Although this hypothesis generally has been supported by results from previous investigations, these studies generally suffer from failure to adjust for possible confounders, such as genetic or early environmental factors, and sometimes also from limited power or low range of exposure. Twin studies give ideal opportunities to disentangle the associations between birth characteristics and diseases with regard to intrauterine environment, genetic factors and environment early in life. We are augmenting the information in the registry by retrieving information on birth characteristics from birth records for all like-sexed twin pairs born in Sweden from 1926-1958.

\section{SALT-II}

The registry has information on exposures and disease outcomes since the 1960s, and as such, provides a valuable resource for prospective studies of risk and protective factors for disease. Nevertheless, for many issues, analysis of incident cases is more desirable than reliance on prevalent cases. Furthermore, information on many diagnoses cannot be extracted by record linkage with the national inpatient registry, as health care is such that fewer cases are treated in circumstances that require an overnight hospital stay. Thus, we plan to repeat the SALT screening procedure in order to ascertain incident cases of most common-complex diseases. To begin with, this effort will focus on twins 65 years and older, as this is the age group in which many diseases have their debut.

\section{Acknowledgments}

The Swedish Twin Registry is funded by grants from the Department of Higher Education, the Swedish Scientific Council, and ASTRA Zeneca. SALT was funded by the Swedish Council for the Planning and Coordination of Research (FRN) and by a grant from the National Institutes of Health (grant AG 08724). SATSA is funded by the National Institutes of Health grants AG 04563 and 10175, OCTO-Twin by AG 08861, HARMONY by AG 08724, the Parkinson's study by ES 10758, Chronic Fatigue Syndrome study by NS41483, and Gender by the MacArthur Foundation Research Network on Successful Aging, FRN, and the Axel and Margaret Ax:son Johnson Foundation.

\section{$\overline{\text { References }}$}

Cederlöf, R. (1966). The twin method in epidemiological studies on chronic diesease. Stockholm, Sweden: Karolinska Institutet.

Cederlöf, R. \& Lorich, U. (1978). The Swedish Twin Registry. In W. E. Nance, G. Allen, \& P. Parisi (Eds.), Twin research: Biology and epidemiology (pp. 189-195). New York: Alan R. Liss.

Eley, T. C., Lichtenstein, P., \& Stevenson, J. (1999). Sex differences in the etiology of aggressive and non-aggressive antisocial behavior: Results from two twin studies. Child Development, 70, 155-168.

Eley, T. C., Lichtenstein, P., \& Stevenson, J. (in press). A longitudinal behavioural genetic analysis of the etiology of aggressive and non-aggressive antisocial behavior. Results from two twin studies. Development and Psychopathology.

Finkel, D., Pedersen, N. L., Plomin, R., \& McClearn, G. E. (1998). Longitudinal and cross-sectional twin data on cognitive abilities in adulthood: The Swedish Adoption/Twin Study of Aging. Developmental Psychology, 34, 1400-1413.

Gatz, M., Pedersen, N. L., Berg, S., Johansson, B., et al. (1997). Heritability for Alzheimer's Disease: The study of dementia in Swedish twins. Journal of Gerontology: Medical Sciences, 52A, M117-M125.

Gold, C. H., Malmberg, B., McClearn, G. E., Pedersen, N. L., \& Berg, S. (2002). Gender and health: A study of older unlike-sex twins. Journal of Gerontology: Social Sciences, 578, 5168-5176.

Heller, D., de Faire, U., Pedersen, N. L., Dahlen, G., \& McClearn, G. E. (1993). Genetic and environmental influences on serum lipid levels in twins. New England Journal of Medicine, 328, $1150-1156$.

Heller, D. A., Pedersen, N. L., de Faire, U., \& McClearn, G. E. (1994). Genetic and environmental correlations among serum lipids and apolipoproteins in elderly twins reared together and apart. The American Journal of Human Genetics, 55, 1255-1267.

Hong, Y., Pedersen, N. L., Brismar, K., \& de Faire, U. (1997). Genetic and environmental architecture of the features of the insulin-resistance syndrome. American Journal of Human Genetics, 60, 143-152.

Hubinette, A., Cnattingius, S., Ekbom, A., DeFaire, U., et al.(2001). Birthweight, early environment, and genetics: A 
study of twins discordant for acute myocardial infarction. Lancet, 357, 1997-2001.

Lichtenstein, P., deFaire, U., Floderus, B., Svartengren, M., Svedberg, P., \& Pederson, N. L. (2002). The Swedish Twin Registry: A unique resource for clinical epidemiological and genetic studies. Journal of Internal Medicine, 252, 1-22.

Lichtenstein, P., \& Annas, P. (2000). Heritability and prevalence of specific fears and phobias in childhood. Journal of Child Psychology and Psychiatry, 41, 927-937.

Lichtenstein, P., Holm, N. V., Verkasalo, P. K., Iliadou, A., Kaprio, J., et al. (2000). Environmental and heritable factors in the causation of cancer - Analyses of cohorts of twins from Sweden, Denmark, and Finland [see comments]. New England Journal of Medicine, 343, 78-85.

Lichtenstein, P., \& Svartengren, M. (1997). Genes, environments, and sex: Factors of importance in atopic diseases in 7-9 year-old twins. Allergy, 52, 1079-1086.

Marenberg, M. E., Risch, N., Berkman, L. F., Floderus, B. \& deFaire, U. (1994). Genetic susceptibility to death from coronary heart disease in a study of twins. New England Journal of Medicine, 330, 1041-1046.

McClearn, G. E., Johansson, B., Berg, S., Pedersen, N. L., et al. (1997). Substantial genetic influence on cognitive abilities in twins 80 or more years old. Science, 276, 1560-1563.
Medlund, P., Cederlöf, R., Floderus-Myrhed, B., Friberg, L., \& Sörensen, S. A (1977). New Swedish Twin Registry. Acta Medica Scandinavica, Suppl., 600.

Pedersen, N. L. (1996). Gerontological behavioral genetics. In J. E. Birren, \& K. W. Schaie (Eds.), Handbook of the psychology of aging (pp. 59-77). San Diego, CA: Academic Press

Pedersen, N. L., Gatz, M., Berg, S., \& Johansson, B. N. (2002). How heritable is Alzheimer's disease late in life? Findings from Swedish twins. Manuscript submitted for publication.

Pedersen, N. L., McClearn, G. E., Plomin, R., Nesselroade, J. R., Berg, S., DeFaire, U., Mortimer, J. A., et al. (1991). The Swedish Adoption Twin Study of Aging: An update. Acta Geneticae Medicae et Gemellologiae (Roma) 40, 7-20.

Pedersen, N. L., Plomin, R., Nesselroade, J. R., \& McClearn, G. E. (1992). A quantitative genetic analysis of cognitive abilities during the second half of the life span. Psychological Science, 3, 346-353.

Reiss, D., Pedersen, N. L., Cederblad, M., Lichtenstein, P., et al. (2001). Genetic probes of three theories of maternal adjustment: II. Genetic and environmental influences. Family Process, 40, 261-272.

Zdravkovic, S., et al. (submitted). Heritability of death from coronary heart disease: 36 years follow-up of 20,966 Swedish twins. 